\title{
INTEGRATING LARGER RES SHARE IN DISTRIBUTION NETWORKS: ADVANCED VOLTAGE CONTROL AND ITS APPLICATION ON REAL MV NETWORKS
}

\author{
Diana MONETA, Paolo MORA, Mario BELOTTI, \\ Claudio CARLINI, Alessandro BOSISIO \\ RSE Ricerca sul Sistema Energetico SpA - Italy \\ \{diana.moneta;paolo.mora;mario.belotti;claudio.carlini; \\ alessandro.bosisio\} @ rse-web.it
}

\author{
Lilia CONSIGLIO
}

ENEL Distribuzione - Italy

lilia.consiglio@enel.com

\begin{abstract}
The paper describes the algorithm that supports increasing the hosting capacity of the network by means of Voltage control of Medium Voltage feeders in presence of storage units. Starting from description of main characteristics and simulation results, the document depicts the future application of the algorithm in a real MV network.
\end{abstract}

\section{INTRODUCTION}

Exploitation of Renewable Energy Resources (RES) represents one of the basic pillars in the complex European strategy aiming at promoting a more sustainable energy production, enhancing energy independence, reducing pollution, and, eventually, stimulating a step forward for technology innovation. It's worth underlining that in most EU countries the reaching of the so-called '20-20-20' targets will require a stronger contribution from the electricity sector to counterbalance expected lesser results from transportation and building sectors. New RES plants, with the major exception of large dam hydroelectric plants and wind parks, will be likely connected to distribution networks. That said, diffusion of Distributed Generation (DG) requires new strategies to ensure reliable and economic operation: large RES share entails well known possible criticalities for the power system [1].

\section{NEEDS FOR ADVANCED CONTROL FUNCTIONALITIES}

In the next future, even with a strong increasing of third party actors in the system, it is likely that Distribution System Operators (DSO) will maintain their central role regarding the regulation operation of the electrical network. In particular, given a network, the DSO should continue guarantee adequate quality parameters regarding the energy delivery to its customers (both passive and active) and a safe operation of the infrastructure. On the other hand, in order to foster RES diffusion, DSOs should accept applications for new DG plants, according to connection criteria established in each country.

Furthermore, supplementary constraints are deriving from the transmission system and will be likely included in grid codes in the next future. Together with more detailed information about actual load and generation combined profiles, maximum counter-flow and minimum allowed power factor in the power exchange with the HV network will be requested.

As a result of those conflicting drivers, DG diffusion could be limited unless more advanced control approaches become admissible. Both DSO and DG units should change their current roles; a stronger co-participation will result at last in a higher presence of DG itself (increase of the hosting capacity), towards the 'active distribution network' concept [2].

To address the above mentioned issues, RSE is developing an algorithm (named "DISCoVER" in the following) that supports increasing the hosting capacity of the network by means of Voltage control of Medium Voltage feeders in presence of storage units.

\section{OPTIMIZATION PROCEDURE}

The goal of the procedure is to identify an admissible condition for a MV network in presence of Distribute Generation, with the minimum dispatching cost for the DSO.

Starting from forecasted load and generation, and considering technical constraints and dispatching costs for active and for reactive power, the proposed algorithm for each time period estimates the state of the system and, when necessary, generates a set of commands for controllable resources that guarantees achievement of technical goals minimizing the overall dispatching cost. In few words, the algorithm searches the minimum of the cost function that respect the following constrains:

- Voltage at nodes within allowed range

- Current in branches within allowed range

- P, Q capabilities of controllable resources

- Energy capabilities of storage unit(s).

The overall cost function is given by the sum of different terms, regarding the requested displacement of controllable resources:

- OLTC transformers and capacitor banks, which are directly operated by DSO;

- reactive power injection/absorption from controllable third-party resources (sub-set of DERs);

- active power from controllable third-party resources (sub-set of DERs);

- energy in storage unit(s), directly operated by the DSO.

The algorithm may be employed in a off-line approach, as described in the present document, or included in a centralized on-line controller, located basically at the primary substation (see Future evolutions). 
In the following, a description of constraints and their mathematical handling is reported, while the algorithm details are given in the references.

\section{Ancillary services \& Business models}

It becomes clear that identification of adequate business models (i.e. regulation scenarios) regarding DSO control capabilities and relevant rewards for ancillary services offered by different resources will play a crucial role in the diffusion of active distribution networks. Costs of internal resources and rewards for controllable generators are included in the optimization algorithm according to different scenarios, in order to explore their impacts on possible control performances.

Firstly, ancillary services may be offered according to a voluntary or a mandatory scheme (the latter for bigger generators, reasonably). Secondarily, several reward schemes for services offered by DERs are possible:

- Administrated price: fixed price established by the Authority; a differentiation based on generator's technology/size could be applied;

- Market scheme: DER operators fix prices for their services;

- Mixed approach, for example a simplified bidding phase that fixes prices for the entire year.

A dedicated research activity was started to explore how different reward scenarios may affect control capabilities and benefit/cost results. As a key results, a pure 'market scheme' appears not fully realistic for MV networks, since generators' size doesn't justify the creation of a complex structure to bid in a real-time market. A fixed fee seems quite reasonable for a number of reasons: as stated before, for small size generators it could be difficult to manage a complex tariff scheme. Additionally, the algorithm could neglect the 'initial' point where the displacement is calculated from: the fee is given independently from the actual power that would be generated by the plant without the requested modulation (quite difficult to be estimated for wind and solar plants especially). Conversely, it implies a mixed-integer approach: in this version of the algorithm only fees proportional to the displacement from an estimated / scheduled initial point are handled, while in the next version fixed rewards will be considered, too. In any case, the algorithm here described allows to specify different rewards for each resource, even on a hourly basis. At this state, the 'fees' should be considered not as actual compensations but as a method to rank regulation resources and their operational costs.

\section{Mathematical approach}

The presence of storage unit(s) requires a complex solving approach: unlike devices placed near RES plants (just to smooth their generation profile) and managed by their owners, a storage unit directly operated by the DSO implies that the algorithm has to calculate how and when to recharge it. This involves to resolve simultaneously more temporal intervals with an integral constraints in the optimization problem. Each storage unit [3] is considered in a single time period as a generator or as a load; efficiencies in charging and discharging processes are taken into account thanks to two "virtual" lines with different electric parameters connecting the real node with a virtual battery node. In order to include integral constraint deriving from storage units, time 'sections' are converted into spatial interconnected sections [4] and the resultant optimization problem is then solved simultaneously for the entire time horizon.

Constraints regarding the net power exchange with the HV network are managed considering the HV node itself as a 'generator' with specific costs for allowing or conversely limiting $\mathrm{P}$ and $\mathrm{Q}$ power counter flow (from MV to HV), according to an hourly scheduling.

In order to establish the control possibilities, the algorithm must know the capability curve of each dispatchable resource; in this analysis the "rectangle" simplification was assumed (see Figure 1).

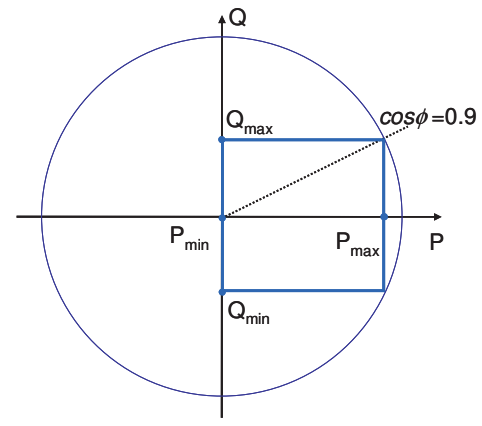

Fig. 1: example of simplified capability curve (photovoltaic generator).

In a given time period, DISCoVER splits each controllable resource as three different sources: a fixed point $\left(\mathrm{SP}_{\mathrm{e}}\right)$, that is the starting estimated point, one "generator" $\left(G_{P}\right)$ and one "load" $\left(\mathrm{G}_{\mathrm{C}}\right)$ with reference to the capability limits as above detailed and reported in Figure 1. Regarding the reactive power, $\mathrm{G}_{\mathrm{P}}$ can assume only positive $Q$ displacements while $G_{C}$ only negative ones.

From $\mathrm{SP}_{\mathrm{e}}$, DISCoVER then can calculate the total $\Delta \mathrm{P}$ (increase or decrease), $\Delta \mathrm{Q}$ (reactive or inductive) and relevant cost for each displacement from the starting point and can search the minimum for the total cost [5]. Finally, a routine in the general algorithm provides a conversion of calculated $\Delta \mathrm{P}, \Delta \mathrm{Q}$ to real set points.

\section{SIMULATION SCENARIOS}

The initial test networks are not representative of a real case but they were designed just to explore different scenarios and to test the algorithm effectiveness. Given a network, several scenarios were built mixing specific parameters:

- number and position of controllable resources 
(generators and storage unit),

- storage technologies (i.e its capability curve and costs),

- load and generation profiles,

- rewards for ancillary services:

- modulation of reactive power (all generators),

- modulation of active power (with respect to their specific technology).

At least 30 different combinations were explored, in order to analyse the dependence of the solution on a specific parameter. The following scheme represents one test network with several generators and one storage unit.

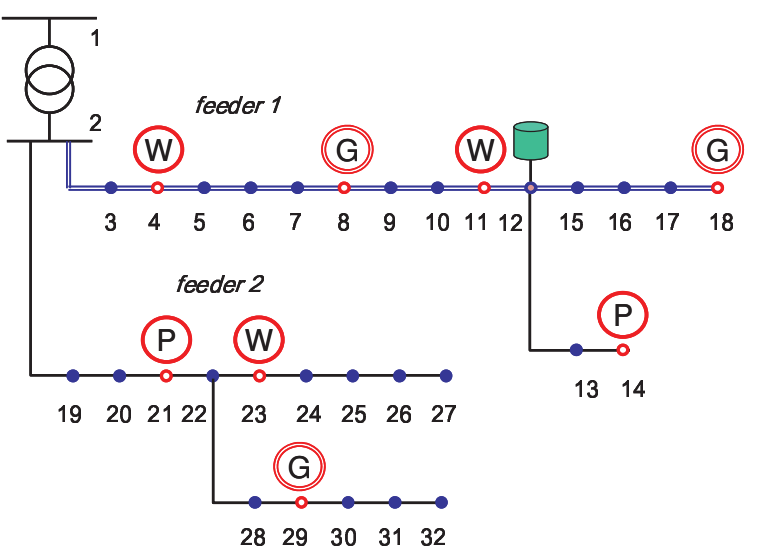

Fig 2. Test network. $\mathrm{W}=$ wind generator, $\mathrm{P}=$ photovoltaic plant, $\mathrm{G}=$ sync. Generator (controllable). Storage unit is connected to node \#12.

\section{Example}

Generation and load profiles were set in order to get feeder 1 as 'passive' (load absorption greater than local generation) and feeder 2 as 'active', that is a condition where OLTC cannot contribute effectively to the optimization. The proposed scenario may represent a condition where PV plants spread unevenly in sub-urban areas. The following graph depicts load and generation profiles before the optimization. In certain periods, for example in \#16 (see Figure 4), an overvoltage is expected in feeder 2 while feeder 1 is in undervoltage. The allowed range was set to $\pm 6 \%$ of rated voltage in order to force the storage unit to be used ahead of third-party generators.

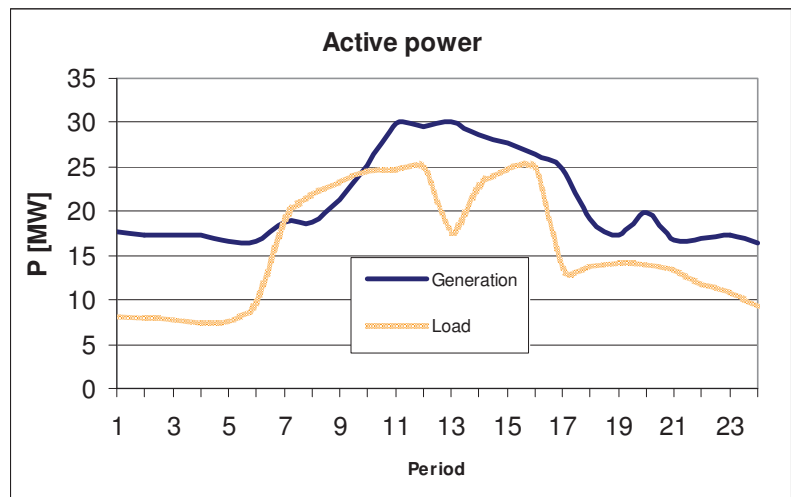

Fig. 3. Scenario 3 (unbalanced feeders), total active power before the optimization.

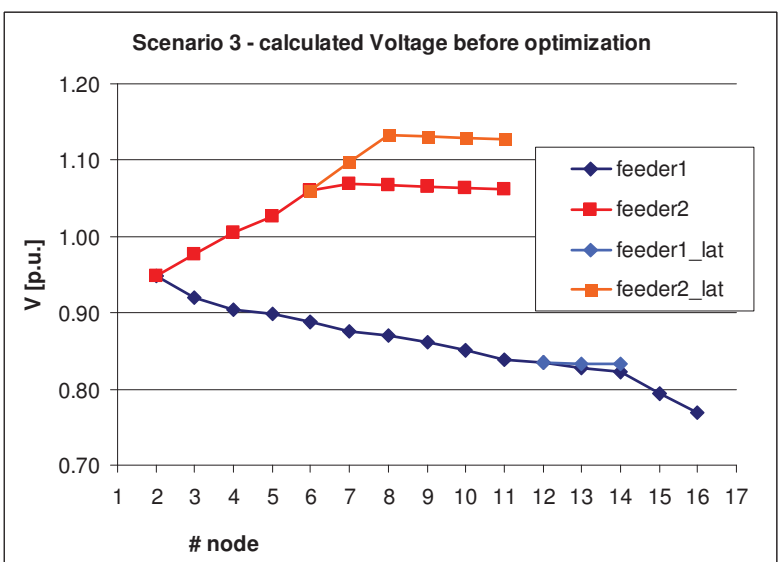

Fig. 4. Calculated voltages before the optimization - period \#16.

The optimization was carried out considering a negligible cost for the Q modulation, and higher costs (comparable to wholesale energy prices) for the modulation of active power - by synchronous generators only. The following graph shows the calculated pattern for the storage unit, with a constraint on the final energy level (50\%, equal to $3 \mathrm{MWh}$ ). For the period \#16 in particular, the Table I summarizes the calculated set-points for the controllable resources (storage and synchronous generators).

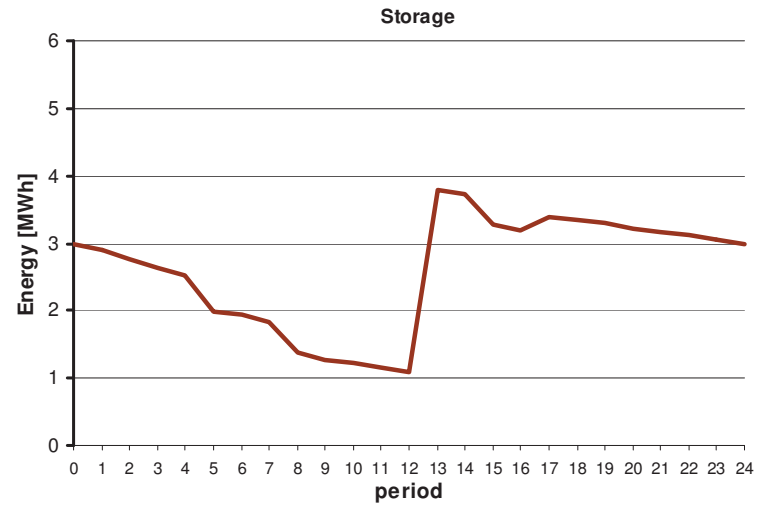

Fig. 5. Calculated energy profile for the storage unit (3 MW / $6 \mathrm{MWh}$, allowed energy range is restricted to $1 \div 4 \mathrm{MWh}$ ).

Thanks to reactive power absorption in feeder 1 , and a combination of active power curtailment and reactive power injection in feeder 2 , it was then possible to reduce the 'fork' between the voltage profiles. The OLTC position is then set down for 2 steps to adjust the overall voltages (higher MV busbar voltage). The Figure 6 shows the voltages profile at period \#16 calculated by the algorithm; it is worth underlining that the constraint on net counterflow towards the HV level played the main role in the curtailment of the active power generation

Table I. Period 16 calculated setpoints for controllable resources:

\begin{tabular}{|c|l|c|c|}
\hline & node & $\Delta \mathbf{P}[\mathbf{M W}]$ & $\Delta \mathbf{Q}[\mathbf{M V a r}]$ \\
\hline \multirow{3}{*}{ Feeder 1 } & $\# 8$ & -1.33 & $\mathbf{- 2 . 2 1}$ \\
\cline { 2 - 4 } & $\# 18$ & 0 & 0 \\
\cline { 2 - 4 } & $\# 12$ (storage) & $0.1[\mathrm{MWh} / \mathrm{h}]$ & $\mathbf{- 0 . 1 6}[\mathrm{MVarh} / \mathrm{h}]$ \\
\hline Feeder 2 & $\# 29$ & -4.51 & $\mathbf{0 . 1 2}$ \\
\hline
\end{tabular}




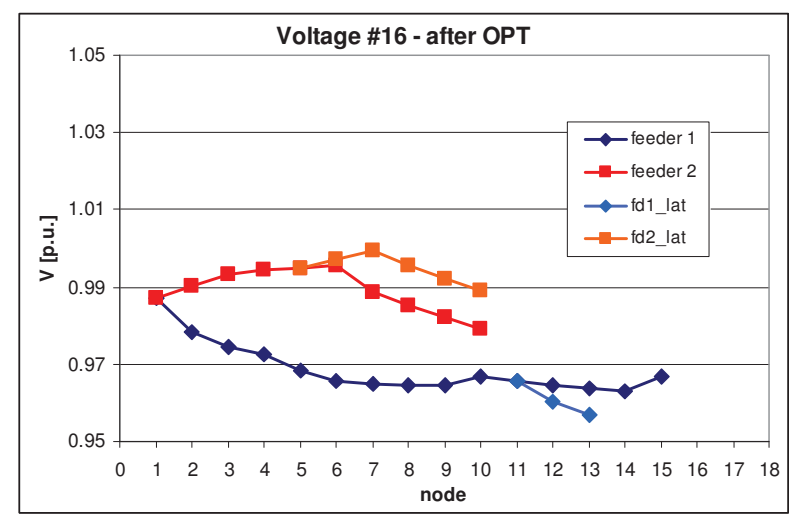

Fig. 6. Unbalanced feeders, voltage profiles after optimization.

\section{FUTURE EVOLUTION: THE GRID4EU PROJECT}

The goal of the project "Large Scale Demonstration of Advanced Smart Grid solutions with wide Replication and Scalability potential for Europe" (GRID4EU) is to carry on demonstration pilots of Smart Grids solutions on a large scale basis. The project involves 27 partners in 12 EU countries; it is coordinated by ERDF, the main French distribution company, and has its technical management belonging to ENEL. The initiative will implement 6 demonstration projects, with the objective of sharing experiences and results in order to give general indication for development of future smart grids. It proposes solutions that go beyond the existing limits for electricity networks through the large scale integration of distributed generation, the improvement of energy efficiency, the enabling and integration of active demand and new electricity uses.

The ENEL Group's Distribution Network operator is launching an 8.2 million euro demo smart grid project in the Forlì-Cesena area, Emilia Romagna region. The demo project, led by ENEL and implemented with CISCO, RSE, SELTA and SIEMENS as partners, will also benefit from EU financial contribution.

The core of the project is an advanced control system, which will communicate through a broadband communication infrastructure with the renewable generators and with all the relevant facilities of the medium voltage network, including storage device(s).

The controller will be based on the algorithms described in the previous paragraph: the resulting system will therefore be able to control power flows and voltage levels to properly integrate distributed generation in the network, as well as testing different solutions for energy storage.

The project will involve two primary substations, around 30 medium voltage lines, approximately 160 medium voltage/low voltage substations and at least five distributed generation facilities. The five facilities, for a total installed capacity of around $2 \mathrm{MW}$, are located in the Forlì-Cesena area. Furthermore, ENEL Distribuzione has ongoing requests for connecting further $13 \mathrm{MW}$ of generation capacity in that area. Overall, 45,000 low voltage customers will benefit from the project's implementation, even though they will not be directly involved in the experimentation.

\section{FINAL CONSIDERATIONS}

This paper presented a centralized algorithm for voltage control of an active distribution network relying on several regulation resources. Unlike local controllers that may face overvoltages caused by diffuse generators, the proposed approach could be necessary to meet additional constraints regarding the network on the whole, for example limitation in the net power exchange with the HV level.

The algorithm will be improved in order to include more complex capability curves, to allow handling of complex constraint about the exchange with the HV level, and to include fixed fee rewards for ancillary services. Above all, a specialized version of the controller will be tested on a real MV network within the GRID4EU project.

\section{ACKNOWLEDGMENTS}

This work has been financed by the Research Fund for the Italian Electrical System under the Contract Agreement between RSE (formerly known as ERSE) and the Ministry of Economic Development - General Directorate for Nuclear Energy, Renewable Energy and Energy Efficiency stipulated on July 29th, 2009 in compliance with the Decree of March 19th, 2009..

\section{REFERENCES}

[1] J.A. Pecas Lopes et al., 2007, "Integrating distributed generation into electric power systems: A review of drivers, challenges and opportunities", Electric Power Systems Research 77 (2007) 1189-1203.

[2] CIGRE WG C6.11, "Active Distribution Networks: general features, present status of implementation and operation practices", Electra n ${ }^{\circ} 246$, October 2009.

[3] RSE, "L'accumulo di energia elettrica", RSEview monography, 2011 (in Italian).

[4] A. Garzillo, M. Innorta, M. Ricci, "The problem of the active and reactive optimum power dispatching solved by utilizing a primal-dual interior point method", Electric Power \& Energy System, Vol. 20 No. 6, pp. 427-434, 1998.

[5] D. Moneta et al., 2011, "Storage Units: Possible Improvements for Voltage Control of MV Distribution Networks", Proceedings of the 17th Power Systems Computation Conference, Stockholm. 